\title{
BASES TEÓRICAS DE LA INFORMÁTICA JURÍDICA
}

\author{
Ricardo A. Guibourg \\ Universidad de Buenos Aires (Argentina)
}

\section{EI Dr. Deep Blue ${ }^{1}$}

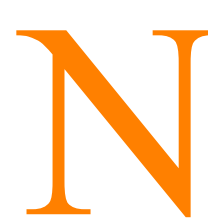

o hace aún veinte años, se instaló en Buenos Aires el primer sistema de informática de gestión, en la mesa de entradas de la Justicia Civil. Cuando ya se hallaba en pleno funcionamiento, un alto magistrado que pasaba por allí se preguntaba, con tono de fastidio: «¿cuándo sacarán todo esto de aquí?». Tal vez sea posible reconstruir el razonamiento subyacente a aquella exclamación. Acaso su autor pensaba que la informática jurídica era un experimento destinado al fracaso, un gasto inútil de espacio, de dinero y de esfuerzo y, lo que es peor, un proyecto que entraba en conflicto con la esencia misma de lo jurídico.

A partir de aquella reconstrucción, dos actitudes frente al derecho pueden distinguirse. Una de ellas es la que trata de aprovechar la tecnología, no sólo para hacer lo mismo de siempre con mayor rapidez sino para potenciar la calidad de lo que se hace, aun al riesgo de tornarlo irreconocible. La otra actitud parte de una concepción estática de la actividad jurídica y sólo admite (tardíamente) los avances tecnológicos cuando se le garantiza que ninguna modificación de fondo habrá de introducirse en el milenario oficio del jurista.

Por supuesto, la informática ha sido hasta ahora incapaz de dar esa garantía. Aun cuando la función de la computadora pueda circunscribirse de hecho a la de una perfeccionada máquina de escribir, sus otras funciones reales o potenciales se hallan a la vista de todos, de modo que es inevitable que tar-

${ }^{1}$ El hombre ha sido siempre celoso de lo que entiende como su superioridad. Luego de haber perdido sus batallas unilaterales con la astronomía, con la zoología y hasta con la psicología, después de haber intentado (afortunadamente sin éxito duradero) segregar a las mujeres, a los indios, a los judíos y a los negros, trataba hace un cuarto de siglo de diferenciarse de las computadoras. Una computadora, se decía, puede ser instruida acerca del movimiento de las piezas del ajedrez; pero su habilidad no pasa de la de un principiante. Este mismo año, una máquina llamada Deep Blue venció a un gran maestro del ajedrez. Muchos lo lamentaron como una derrota del hombre como oponente de la máquina. Otros lo festejaron como un triunfo del hombre, en su calidad de constructor de la máquina. Pero la actitud que escojamos en este tema no cambia un ápice la realidad. 
de o temprano tiendan a aprovecharse. Y, por cierto, la mayoría de esas funciones tiene presupuestos o requisitos que corroen la concepción tradicional.

Algunos piensan que el conflicto con el modo humano de pensar el derecho proviene del extraordinario desarrollo de la inteligencia artificial, y ven en este hecho una forma de rivalidad entre el hombre y la máquina. Sin embargo, parece más cercano a la realidad razonar exactamente a la inversa. Si -por vía de hipótesis- tomamos la inteligencia humana como paradigma del pensamiento, observaremos que la inteligencia artificial carece, al menos por ahora, de la capacidad de aprender por vía analógica y hasta subliminal en la magnitud en que el ser humano puede hacerlo. Si la máquina fuera perfecta (en el sentido definido), bastaría con enviarla a la Universidad y ponerla en contacto con ricas bibliotecas para convertirla en un jurista avezado. Aprendería a atribuir relevancia a lo conocido y repetido, así como a menospreciar las hipótesis nunca planteadas; se habituaría a aceptar opiniones que se postulan como verdaderas sin controlar su consistencia interna ni su deducibilidad de los axiomas generales. Y aceptaría estos axiomas acaso induciéndolos por su cuenta de la conducta espontánea de sus maestros, sin ponerlos en duda hasta que alguna catástrofe la obligase a hacerlo. En otras palabras, el ordenador actuaría como suelen hacerlo juristas y abogados y debatiría con ellos en los mismo términos en los que se desarrollan habitualmente los congresos y simposios.

Pero las máquinas no son tan desarrolladas. Ellas requieren programas en los que cada paso de razonamiento esté explícitamente indicado; procesan datos expresados en forma inequívoca y, finalmente, ofrecen resultados lógicamente deducibles de unos y de otros. Programar una computadora, entonces, no es tarea sencilla para un experto en derecho, habituado al lenguaje vago, a los criterios no explícitos, a cierto casuismo incontrolado y a la aceptación de resultados en cierto modo aleatorios.

Este es el motivo por el que, si el derecho informático es una parte del derecho vigente y si la informática jurídica es una técnica aplicable en principio a cualquier segmento del derecho, las bases teóricas de la informática jurídica constituyen un tema específico de la epistemología del derecho, estrechamente emparentado (y, como sucede en las familias, a veces en conflicto) con la filosofía jurídica.

\section{Enfoques paralelos, desarrollos convergentes}

Julia Barragán, en su reciente artículo para la Enciclopedia Iberoamericana de Filosofía ${ }^{2}$, distingue para la informática jurídica dos enfoques: los

${ }^{2}$ Barragán, Julia, Informática jurídica, Enciclopedia Iberoamericana de Filosofia, volumen 11, El Derecho y la Justicia (de. Ernesto Garzón Valdés y Francisco J. Laporta), Madrid, Trotta, 1996, página 39. 
que denomina como «dominantemente computacional» y de «equilibrio entre las esferas». El primero encara al derecho como un mero campo de aplicación de la informática y busca «algoritmos que se aplican sin restricciones sobre una masa de datos sin forma». En este sentido, los juristas, «como resultado del pecado original de expresarse en su propio lenguaje, y de razonar conforme a sus propios patrones, parecen condenados a no disfrutar nunca plenamente de todas las maravillas que la Informática despliega ante sus ojos asombrados». En el segundo enfoque, en cambio, «la ciencia de la Computación deja de ser el referente inamovible cuya certeza no se pone en duda, para convertirse en un territorio permanentemente sujeto a críticas, refinamientos y validaciones, los cuales derivan necesariamente de la esfera jurídica» y se vinculan «con la necesidad de producir una transformación del entorno en el que se producen las inferencias y las decisiones jurídicas, con la finalidad de que las mismas posean la mejor base argumental alcanzable». En este contexto, las características conocidas del lenguaje del derecho («la textura abierta, el carácter vago de algunas palabras y, fundamentalmente, su semántica») «no son defectos o errores que hay que corregir a cualquier precio para llegar a perfilar un buen conjunto de aplicación de los algoritmos, sino que ellos mismos constituyen condiciones que generan un poderoso estímulo en la definición de estructuras de datos interesantes y complejas, semánticamente conformadas desde un punto de vista jurídico». Destaca la misma autora que la «posibilidad de que exista más de una respuesta correcta, según sean los antecedentes que se seleccionen para tomar la decisión (...) no constituye una herejía que haya que exorcizar rápidamente, sino que siendo el rasgo típico de lo que se conoce como interpretación del Derecho, debe ser preservado en la estructura de los datos».

Sería interesante investigar si aquellos dos enfoques son independientes e incompatibles entre sí o si, por el contrario, su propia dinámica los conduce a unirse a medida que sus presupuestos se clarifiquen y sus objetivos adquieran compatibilidad mutua.

En el Congreso Mundial de 1995, Danièle Bourcier formuló una advertencia: «escribir un sistema experto es codificar el derecho» ${ }^{3}$. Y explicaba que «tanto los modos de elaboración de los sistemas de información legal, como la codificación, forman parte de los modos de racionalización de la producción jurídica», ya sea en el momento de la redacción de los textos o en el de su aplicación. Según Bourcier, los dos instrumentos deben ser co-

${ }^{3}$ Bourcier, Danièle, L'informatisation du droit; réflexions sur l'évolution des techniques d'écriture de l'état, en 17th IVR World Congress, Bologna, 16-21 June, 1995, Challenges to Law at the End of the 20th Century, Bolonia, IVR, 1995, tomo II, página 65. 
herentes (concentración del material jurídico), manejables (incluir únicamente disposiciones normativas), tener una estructura lógica, estar escritos en un lenguaje claro, suprimir las dificultades de interpretación y, finalmente, ser completos en relación con la materia tratada.

A primera vista, las reflexiones de Barragán y de Bourcier parecen referirse a problemas distintos. Mi propósito es mostrar la relevancia que tiene, para el desarrollo del pensamiento jurídico, advertir el punto en que ellas se entrecruzan y confrontan distintas maneras de plantear el mismo problema. Ese punto es el examen (la reconstrucción) de la estructura del pensamiento jurídico.

\section{Las condiciones para el uso de la informática}

Dentro de la informática jurídica, es común distinguir tres campos, en orden creciente de complejidad y, a la vez, en orden cronológico de aparición: la informática documental, la de gestión y la decisoria. La primera fue recibida de buen grado por abogados y juristas. La segunda generó alguna resistencia, que finalmente cedió ante las ventajas que ella ofrecía. La tercera sigue erizando la piel de los juristas y provoca todavía polémicas encendidas. Pero todas ellas, es preciso señalarlo, constituyen distintas maneras, diferentes intensidades en el cumplimiento de una misma función, a saber, la de recibir informaciones, elaborarlas de acuerdo con un programa y ofrecer el producto de tal elaboración al hombre de derecho ${ }^{4}$. Las diferencias, meramente cuantitativas, residen en el grado de complejidad de los criterios de elaboración contenidos en el programa. En todos los casos, sin embargo, la calidad del servicio prestado por la computadora depende, en el derecho como en otros campos de la actividad humana, de dos factores: la representación del conocimiento y el motor inferencial.

El motor inferencial no es otra cosa que la lógica que permite pasar de las premisas a las conclusiones. En este aspecto, el material jurídico ofrece algunas dificultades específicas: es preciso desarrollar una lógica que dé cuenta de ciertas constantes normativas, como obligación, prohibición, facultamiento, acción, omisión, delegación, competencia. La lógica deóntica busca, desde mediados del siglo, dar respuesta a estas necesidades. Aunque queda todavía un buen trecho por recorrer, ya es posible reconstruir formalmente el contenido y el resultado de normas dictadas sucesivamente en un mismo orden normativo ${ }^{5}$.

${ }^{4}$ Esta idea, como muchas de las que siguen, está tomada de Guibourg, Ricardo A., Alende, Jorge O., y Campanella, Elena M., Manual de Informática Jurídica, Buenos Aires, Astrea, 1996.

${ }^{5}$ Una tentativa en este sentido se halla contenida en Guibourg, Ricardo A., Formalization of Competence, en Normative Systems in Legal and Moral Theory, Festschrift for Carlos E. Al- 
La representación del conocimiento, a su vez, es la técnica con la que se describe el segmento del universo que se desea manejar. La realidad geográfica, para usar un ejemplo clásico, puede describirse (modelizarse) mediante mapas; la química con fórmulas, la economía con cifras y gráficos de curvas. ¿Y la jurídica? Los tratados de derecho emplean una forma de representación, pero es dudoso que esa técnica facilite la construcción de sistemas expertos no humanos, ya que emplea conceptos vagos, ideas implícitas y palabras con fuerte contenido emotivo. El texto mismo de las leyes adolece de características similares y requiere una constante tarea de interpretación, sin la cual el derecho no podría ser descripto. Cabe decir, pues, que la representación del conocimiento es un verdadero problema de la informática jurídica que, con diverso grado de dramaticidad, se plantea en todas sus ramas y, en el caso límite de la informática decisoria, se centra en lo que puede llamarse la formalización del razonamiento jurídico.

En efecto, el razonamiento ha de ser adaptado a las necesidades de los sistemas informáticos; pero estas necesidades no son cualitativamente diferentes de las que rigen el pensamiento humano. Semejante adaptación es una suerte de formalización: nada en ella puede quedar sujeto a la improvisación del momento ni librado a la acción de variables ignotas (a menos, claro está, que tales variables sean halladas, formalizadas e introducidas en el programa con motivo del caso particular). Las posibilidades del sistema pueden ser infinitas, como son infinitas la sucesión de los números naturales o las consecuencias de un conjunto dado de premisas; pero todas ellas deben hallarse contenidas en formas o clases de combinaciones de variables que el sistema acepte como relevantes, y aun estas variables han de derivar de un número finito de elementos primitivos, combinables entre sí según aquellas formas.

\section{Deshumanización o clarificación}

La exigencia de adaptar el razonamiento jurídico a las necesidades informáticas es a menudo recibida como una propuesta de deshumanizar el derecho. Los juristas están orgullosos del modo de pensar que cultivan desde hace milenios y, más aún, lo consideran un verdadero bastión de humanismo, apropiado para tratar con valores igualmente humanos: la vida, la libertad, el honor, los derechos en general. En este contexto, el carácter difuso de los criterios de apreciación de la prueba y la textura abierta de las normas, que abre camino a diversas y cambiantes interpretaciones, son canales

chourrón and Eugenio Bulygin (ed. Garzón Valdés y otros), Berlín, Duncker \& Humblot, 1997, página 455. 
por los que el sistema jurídico, formal y frío, recibe la necesaria y constante inyección de equidad. ${ }^{6}$

No concuerdo en absoluto con la actitud que subyace a aquella visión del problema. Los mismos dones de racionalidad con los que el hombre pretende diferenciarse del resto de la escala zoológica terminan a veces por espantarlo cuando se le presentan como asignatura pendiente ${ }^{7}$; pero la evolución del pensamiento humano (es decir, su cambio favorable según sus propios criterios generales de preferencia) tiende invariablemente a poner de manifiesto lo implícito, a clarificar lo que se presenta a la conciencia de un modo confuso y, una vez hecho eso, a concebir nuevas combinaciones de ideas y criterios que, a su vez, serán más tarde objeto del mismo proceso.

La aplicabilidad de esa reflexión al campo del derecho se advierte fácilmente cuando se analiza el modo en que el jurista (abogado, juez, funcionario, observador experto) razona frente a un caso particular. El jurista siente que puede ejercer cierta libertad al emitir su decisión, opinión o dictamen; pero sabe también que su actitud requiere fundamentos, y que no cualquier fundamento (un tiro de moneda a cara o cruz, por ejemplo) es aceptable. El universo de los fundamentos aceptables constituye, en cierto modo, el programa al que el jurista se halla sujeto, integrado por criterios que permiten: a) seleccionar y valorar las pruebas, para decidir a partir de ellas los hechos controvertidos; b) seleccionar, entre estos hechos, los que hayan de considerarse relevantes para caracterizar el caso en examen; c) construir, a partir de estas características relevantes, un esquema del caso que lleve a subsumirlo en un tipo general y d) vincular este tipo general con una solución normativa, también genérica, que después será adaptada a los datos particulares del caso.

La fuente más obvia de estos criterios es la ley. Las normas jurídicas, en sus distintos niveles, proveen al jurista una programación general, frente a la cual éste goza de escasa libertad: su apartamiento de tales criterios, salvo casos excepcionales, como los de inconstitucionalidad, se considera equivocación del abogado o mal desempeño de la función judicial o administrativa.

Otra fuente son los precedentes o la jurisprudencia, conjunto de decisiones anteriores que ejercen sobre el jurista cierta presión para el empleo de criterios interpretativos aunque, según sea el sistema jurídico del que se trate, le dejan mayor amplitud de decisión personal.

${ }^{6}$ Acerca de la equidad como conjunto de criterios no explícitos, me he extendido en Guibourg, Ricardo A., El fenómeno normativo, Buenos Aires, Astrea, 1987, página 118.

${ }^{7}$ Isaac Asimov ha mostrado magistralmente este punto en su cuento ¿Qué es el hombre?, en El hombre del bicentenario, Barcelona, Martínez Roca, 1978. 
Pero estas decisiones personales, tal como las interpretaciones de la jurisprudencia y aun, en buena medida, la resolución de aplicar normas sobre inconstitucionalidad, abuso del derecho o lagunas, dependen a su vez de otros criterios. Sólo que estos criterios no son completa ni predominantemente provistos por la ley ni por los precedentes: se encuentran en la formación moral y profesional del juez, del abogado o del funcionario.

Cada jurista, en efecto, ha resuelto alguna vez su posición frente a ciertos problemas jurídicos o situaciones de hecho, o elegido entre dos o más corrientes jurisprudenciales la que él considera más justa o adecuada; y, si su posición no ha de obedecer a una repentina improvisación, se encuentra en condiciones de explicar los motivos o argumentos que lo conducen a la conclusión escogida.

Pero, si se examinan a fondo esos argumentos, podrá casi siempre advertirse que ellos se basan en conceder a ciertas circunstancias un valor más relevante que el que les otorgan las razones esgrimidas por la argumentación opuesta. Estas diferencias de apreciación -a menudo matices no expresados- son muchas veces desconocidas por el propio jurista que las sustenta: ellas corresponden a su programación ignota, a la que fue absorbida desde la infancia y modelada por un sinnúmero de contingencias personales ocurridas durante su vida, tal vez hoy olvidadas. El fruto de estas incontables e incontrolables condiciones, de su combinación y de su entrechoque durante muchos años en la caja negra del inconsciente, es un individuo autoritario o liberal, más inclinado hacia la derecha o hacia la izquierda, apático o voluntarioso, conformista, temerario o cobarde $\mathrm{y}$, en definitiva, dispuesto -acaso sin saberlo- a reaccionar de cierta manera frente a ciertos estímulos que se le propongan.

Este panorama tan aleatorio -y, mirado de cerca, tan poco alentador- es precisamente el que se encuentra en el fondo de la función judicial o administrativa, el que muchos juristas reivindican y alaban como el indispensable control humano de la aplicación de la ley en cada caso individual, el que se nos muestra glorificado bajo el nombre de equidad.

$\mathrm{Y}$, sin embargo, algo hay de cierto en esa consideración. La aplicación humana de criterios implícitos e inconscientes es la base última de todas las preferencias, y resulta indispensable al derecho, pero no en tributo a su excelso carácter humano, sino a causa de su propia imperfección.

En efecto, todo criterio conocido puede expresarse en palabras y, en caso necesario, llevarse al grado conveniente de precisión. Un criterio expresable y preciso puede formalizarse, ya sea como algoritmo, ya sea como la configuración de un diagrama arborescente del flujo de decisiones. Y un criterio formalizable de esta manera puede ser introducido en el programa de un ordenador, que lo aplicará con mayor rapidez, eficiencia, seguridad e im- 
parcialidad que el propio ser humano. Los criterios desconocidos, en cambio, se encuentran todavía encerrados en la caja negra de nuestra mente, de donde es posible extraerlos en parte por medio de la introspección y del razonamiento o inducirlos por medios estadísticos. Una vez sacados a la luz pueden seguir el camino de los otros; pero mientras eso no ocurra, sólo el hombre puede aplicarlos, ya que forman parte de su programa, sin que existan (todavía) diagramas para reproducirlos ni una vía adecuada para transmitirlos. ${ }^{8}$

\section{Entre el arte y la exactitud}

Para el hombre, pues, una cosa es saber hacer algo (ser capaz de hacerlo en la práctica) y otra diferente saber cómo se hace algo (ser capaz de explicitar los pasos y criterios que, debidamente empleados, conduzcan con seguridad al fin deseado).

Un experto sabe hacer su trabajo. Pero el creador de un sistema experto debe saber cómo se hace ese mismo trabajo, lo que no es lo mismo, e implica por su parte tomas de conciencia y desacralizaciones tal vez dolorosas. Miguel Ángel habría sabido describir la técnica empleada para pintar la Capilla Sixtina, pero se habría sentido perplejo y hasta ofendido por alguien que le preguntase por el minucioso y oscuro proceso intelectual y afectivo que lo había llevado a concebir las imágenes, a elegir los colores, a crear luces o a sugerir transparencias. Esa parte de la «técnica» era desconocida por el propio artista, a la vez que constituía la impronta de su genio. Los artesanos, sin embargo, han condescendido a menudo a analizar su talento en segmentos comprensibles: así el mundo disfruta de la industria. Otro tanto hacen hoy científicos de toda laya y, en unión con los informáticos, dan a luz los sistemas expertos (las actuales máquinas de saber) ${ }^{9}$.

Esta actitud, apenas incipiente en el campo del derecho, es una de las condiciones actuales para el uso de las computadoras. Para la máquina, que no olvida y que carece de subconsciente, saber hacer y saber cómo se hace son una y la misma cosa. Para programarla, es preciso indicarle todos los pasos a seguir en cada situación. Y hacerlo claramente, en un lenguaje que la máquina pueda «comprender». El jurista, habituado a la imprecisión, a las medias palabras, a los argumentos inconcluyentes $\mathrm{y}$, en general, a un método que se parece más al arte que a las matemáticas, emplea y tiene por buenos criterios y datos que no le han sido suministrados formalmente, que no siempre ha controlado racionalmente y que, de hecho, a veces ni siquie-

${ }^{8}$ Guibourg, Ricardo A., El fenómeno normativo (cit.), páginas 145 a 147.

${ }^{9}$ Guibourg, Ricardo A., Sobre la técnica en el derecho, en Guibourg (recopilador), Informática jurídica decisoria, Buenos Aires, Astrea, 1993, páginas 9 y 10. 
ra conoce. Este fondo de criterios ignotos le permite proporcionar soluciones donde las condiciones son vagas o desconocidas, interpretar normas de contenido ambiguo y, en general, adaptar los criterios formales a lo que él entiende como las necesidades del caso particular (esto es, modificar criterios deficientemente expresados en virtud de actitudes valorativas exentas de control intersubjetivo).

El empleo de la informática, en cambio, nos propone una utopía tecnológica. En su versión extrema, ella consiste en proveer, para cada situación de hecho propuesta, una solución legal única, completamente previsible e inmediata. Parece obvio que, si tal cosa pudiera lograrse, no sólo las prácticas jurídicas se verían profundamente modificadas: la actitud de las personas frente al derecho se tornaría completamente diferente.

En efecto, todo el manejo de las normas y de las circunstancias eventualmente relevantes para fines normativos se halla, desde siempre, adaptado al escaso rigor del pensamiento jurídico. Estamos habituados a pensar que toda decisión de derecho requiere alguna elección entre criterios, ya sea de identificación y apreciación de la prueba, de interpretación de las normas, de la consiguiente subsunción del caso en la norma interpretada $\mathrm{y}$, en algunos casos, de elección discrecional de las consecuencias dentro de un marco provisto por la ley. Esa opción entre criterios diversos no está a su vez determinada por metacriterios unívocos ni públicos, sino por procedimientos y presupuestos que integran el más o menos ignoto sistema de pensamiento del individuo que haya de adoptarla. Esta última circunstancia no nos parece un mal necesario, sino una ventaja inherente a la humanidad de la materia a tratar, y por esto concedemos a los decisores un prestigio social tanto mayor cuanto menor sea la predecibilidad de sus decisiones, así como, en caso de divergencia entre un número plural de decisores, aceptamos el criterio mayoritario con un respeto casi religioso. Como consecuencia, nadie está positivamente seguro de la licitud o de la ilicitud de una conducta determinada hasta que el tribunal de última instancia se ha expedido acerca de ella: lo que llamamos seguridad jurídica es, en el mejor de los casos, un grado más elevado que otros en la probabilidad de acierto de las predicciones que formulamos.

Si la utopía informática se realizara, cada individuo podría saber de inmediato la calificación deóntica de sus conductas y, en caso de llevarlas a cabo, la protección o el castigo que el sistema jurídico hubiese dispuesto para ellas sobrevendría automáticamente, de inmediato y en forma absolutamente previsible. Es fácil imaginar el impacto que esta situación tendría entre deudores y acreedores, abogados y potenciales infractores y, de paso, el modo en que descendería la importancia relativa de jueces y funcionarios para dar paso a una sociedad regida por una ley común, pública y transpa- 
rente, antes que por la mano más o menos generosa de magistrados y funcionarios.

\section{Más acá de la utopía informática}

Desde luego, la utopía informática no es íntegramente realizable. No porque la naturaleza del material a tratar constituya en sí misma un obstáculo, sino porque no existen, al menos por ahora, medios técnicos adecuados para satisfacer algunas exigencias de aquel modelo ni condiciones políticas para emplear consistentemente los medios técnicos disponibles. El modelo de sistema normativo propuesto por Alchourrón y Bulygin ${ }^{10}$, por ejemplo, es una excelente base para reconstruir el orden jurídico de un modo mucho más riguroso que el actual; pero es difícil imaginar a los legisladores dispuestos a diseñar sus leyes mediante matrices de casos. De todos modos, aún en ese supuesto, no menos de dos problemas quedarían en pie. Uno de ellos es el de la quaestio facti: puesto que los hechos relevantes para la configuración de cada caso son a menudo mal conocidos $\mathrm{y}$, en no pocos casos, llevados a cabo en la clandestinidad, no existe todavía una teoría de la prueba capaz de dar cuenta satisfactoriamente de todos los criterios de apreciación que puedan emplearse. El segundo, naturalmente, sigue siendo el de la quaestio iuris: aunque el legislador definiera los casos relevantes con el mayor rigor disponible, el uso de un lenguaje natural dejaría abierto un campo indefinido para el debate acerca de los límites de cada caso tipo y de su aplicabilidad a una situación concreta.

Sin embargo, estas dificultades sólo se oponen al caso extremo de lo que he llamado la utopía informática. Ellas no impiden avanzar indefinidamente por el camino indicado, al menos hasta chocar, en cada caso, con algún obstáculo que las circunstancias no permitan sortear.

En algunas materias, cuando el cálculo político de costos y beneficios parezca justificarlo, es posible aceptar una deshumanización de la prueba: someterla a formas estrictas, preferentemente preconstituidas, tasar su valor... y aceptar los resultados del sistema trazado. Éste es un tema en el que las valoraciones suelen tomarse por limitaciones fácticas. Por ejemplo, cualquier jurista sentiría horror frente a un sistema procesal penal en el que la libre convicción del fiscal, expresada en la acusación, se considerase en sí misma una prueba del delito y el acusado tuviera a su cargo la prueba de su inocencia. Sin embargo, este mismo sistema se halla en vigencia en materia de infracciones de tránsito, donde el acta levantada por el agente de policía sirve a la vez como acusación y prueba ante el tribunal municipal. Que

${ }^{10}$ Cfr. Alchourrón, Carlos E., y Bulygin, Eugenio, Introducción a la metodología de las ciencias jurídicas y sociales, Buenos Aires, Astrea, 1974. 
quede en claro, por supuesto, que no estoy proponiendo universalizar un procedimiento tan dudoso, sino tan sólo sugerir que hay casos (habitualmente menores) en los que la sociedad privilegia la preconstitución de la prueba incluso frente a las garantías de justicia individual ${ }^{11}$.

A la inversa, en la determinación del derecho aplicable es posible mantener una relativa humanización. Cada criterio normativo es acaso como un iceberg, del que la punta conocida puede ser rigurosamente descripta por cualquiera, mientras su base permanece sumergida en un mar de potencialidades subconscientes. Dado que la decisión sobre cada punto desconocido, aunque sea adoptada en un caso individual, implica por sí sola un criterio general para casos semejantes, es posible rescatar de la imprecisión segmentos cada vez mayores de cada sistema de criterios, a medida que los planteos individuales lo hagan necesario o conveniente. Una vez advertidos y hechos explícitos, los criterios pueden ajustarse al consenso público o a la decisión política competente e integrarse a la parte rigurosamente descripta y formalizada ${ }^{12}$.

Si hemos de querer emplear aquellos procedimientos es un interrogante político. También lo es decidir cuándo y en qué casos hemos de emplearlos, o qué precio estamos dispuestos a pagar para obtener las ventajas que de ellos han de resultar. Lo que sostengo, por mi parte, es que es perfectamente posible avanzar por el camino del rigor normativo mucho más que lo que los juristas están normalmente dispuestos a admitir.

En este sentido, la principal base teórica de la informática jurídica consiste en un cambio epistemológico. A la luz de este enfoque, las opiniones citadas al principio se muestran como tomas de posición divergentes ante el problema de la racionalidad en el derecho. Julia Barragán parece expresar conformidad con la orientación epistemológica tradicional: el jurista tiene derecho a su propia forma de razonar, que incluye la textura abierta del lenguaje y desemboca en más de una respuesta correcta para cada problema. Danièle Bourcier, desde una óptica más específica, pone de resalto que el uso de la informática jurídica es, como la codificación, un modo de racionalización de la producción jurídica. La racionalidad, desde luego, es un largo camino que, mediante la clarificación de las premisas y la formalización de las reglas de inferencia, tiende a obtener una sola respuesta para cada problema. Y, si la respuesta no es satisfactoria, aconseja revisar las premisas y controlar el uso de las reglas antes que introducir en el sistema una in-

${ }^{11}$ Propuestas de procedimientos formularios, con aplicación de algunas de estas técnicas, pueden consultarse en Guibourg (recopilador), Informática Jurídica Decisoria (obra citada). siguientes

2 Cfr. Guibourg, Ricardo A., El fenómeno normativo (obra citada), páginas 159 y 
consistencia individual. En lo personal, tiendo a compartir la actitud de Bourcier y observo que muchos de los argumentos que hoy sirven de obstáculos epistemológicos al aprovechamiento de la informática en el derecho se parecen a los que otrora se oponían a la codificación.

\section{Misterio humano, demasiado humano}

Los humanos estamos acostumbrados a aceptar con alegría y hasta con orgullo las imprecisiones de nuestro razonamiento, especialmente en ciencias humanas. La informática nos pone frente a una evidencia y a un desafío que no son sólo jurídicos. Una vía epistemológica, la que venimos usando, es ensalzar el misterio, rechazar la lógica o recortar sus alcances y creer que todos hablamos de lo mismo y colaboramos desde distintas posiciones para desentrañar misterios cambiantes y a la postre insondables. La otra consiste en desmitificar los misterios: no dejar de reconocer nuestra limitada capacidad para lidiar con ellos, pero situarlos en nosotros mismos. El misterio, en efecto, no es otra cosa que el conjunto de nuestros criterios y de las relaciones que entre ellos establecemos. Si nos parece un abismo insondable, es porque nuestra mente contiene agujeros muy profundos. Poco a poco, sin embargo, podemos explorarlos y hacer públicos nuestros hallazgos. Sólo en esos límites podremos entendemos realmente (como lo hacemos en el ámbito de las ciencias naturales), para debatir nuestras preferencias en un marco conocido por todos. El resto será un misterio que nos invite a penetrar en él juntos, para que los pedazos que exploremos dejen de ser misteriosos. Para esto son indispensables los juristas, armados del libre debate y de la lealtad intelectual. Pero, después de todo, esta es la diferencia que siempre conocimos entre lo que sabemos y lo que ignoramos. Una cosa es estudiar y aplicar una ciencia y otra es investigarla. Si cada aplicación de la física ya conocida debiera someterse a debate y experimentación como la física que estamos investigando, las ciencias naturales habrían obtenido hasta ahora resultados tan pobres como las ciencias jurídicas. 